\title{
Distinct genetic difference between the Duffy binding protein (PkDBPall) of Plasmodium knowlesi clinical isolates from North Borneo and Peninsular Malaysia
}

Mun-Yik Fong ${ }^{1,2^{*+}}$, Sarah AA Rashdi ${ }^{1 \dagger}$, Ruhani Yusof ${ }^{1}$ and Yee-Ling Lau ${ }^{1,2}$

\begin{abstract}
Background: Plasmodium knowlesi is one of the monkey malaria parasites that can cause human malaria. The Duffy binding protein of P. knowlesi (PkDBPall) is essential for the parasite's invasion into human and monkey erythrocytes. A previous study on $P$. knowlesi clinical isolates from Peninsular Malaysia reported high level of genetic diversity in the PkDBPall. Furthermore, 36 amino acid haplotypes were identified and these haplotypes could be separated into allele group I and allele group II. In the present study, the PkDBPall of clinical isolates from the Malaysian states of Sarawak and Sabah in North Borneo was investigated, and compared with the PkDBPall of Peninsular Malaysia isolates.

Methods: Blood samples from 28 knowlesi malaria patients were used. These samples were collected between 2011 and 2013 from hospitals in North Borneo. The PkDBPall region of the isolates was amplified by PCR, cloned into Escherichia coli, and sequenced. The genetic diversity, natural selection and phylogenetics of PkDBPall haplotypes were analysed using MEGA5 and DnaSP ver. 5.10.00 programmes.

Results: Forty-nine PkDBPall sequences were obtained. Comparison at the nucleotide level against $P$. knowlesi strain $\mathrm{H}$ as reference sequence revealed 58 synonymous and 102 non-synonymous mutations. Analysis on these mutations showed that PkDBPall was under purifying (negative) selection. At the amino acid level, 38 different PkDBPall haplotypes were identified. Twelve of the 28 blood samples had mixed haplotype infections. Phylogenetic analysis revealed that all the haplotypes were in allele group I, but they formed a sub-group that was distinct from those of Peninsular Malaysia. Wright's $F_{S T}$ fixation index indicated high genetic differentiation between the North Borneo and Peninsular Malaysia haplotypes.

Conclusions: This study is the first to report the genetic diversity and natural selection of PkDBPall of $P$. knowlesi from Borneo Island. The PkDBPall haplotypes found in this study were distinct from those from Peninsular Malaysia. This difference may not be attributed to geographical separation because other genetic markers studied thus far such as the $P$. knowlesi circumsporozoite protein gene and small subunit ribosomal RNA do not display such differentiation. Immune evasion may possibly be the reason for the differentiation.
\end{abstract}

Keywords: Plasmodium knowlesi, North Borneo, Duffy binding protein, Diversity, Selection, Haplotypes, Alle groups

\footnotetext{
* Correspondence: fongmy@um.edu.my

${ }^{\dagger}$ Equal contributors

${ }^{1}$ Department of Parasitology, Faculty of Medicine, University of Malaya, 50603 Kuala Lumpur, Malaysia

${ }^{2}$ Tropical Infectious Diseases Research and Education Centre (TIDREC), Faculty

of Medicine, University of Malaya, 50603 Kuala Lumpur, Malaysia
} 


\section{Background}

Plasmodium knowlesi, a malaria parasite of macaque monkeys, was reported to cause a large number of human infections in the Malaysian state of Sarawak, North Borneo, in 2004 [1]. Subsequent to this landmark report, human knowlesi malaria cases have been documented in in other parts of Borneo Island, Peninsular Malaysia, and in many other countries in Southeast Asia [2]. In Malaysia, P. knowlesi has now overtaken Plasmodium vivax as the main cause of human malaria [3].

The invasion of a malaria parasite into its host erythrocyte depends on the interaction between the parasite's protein and its corresponding receptor on the surface of the erythrocyte. Plasmodium knowlesi uses the Duffy blood group antigen as a receptor to invade erythrocytes [4]. The Duffy binding proteins of P. knowlesi (PkDBP) are located on their merozoites and occur as three distinct forms: $\alpha, \beta$ and $\gamma$. These are large proteins and each can be divided into seven regions (I-VII). Region II contains the critical motifs for binding to the erythrocyte. Region II of PkDBP $\alpha$ (designated as PkDBP $\alpha \mathrm{II}$ ) binds to Duffy-positive human erythrocytes and macaque erythrocytes. PkDBP $\beta I I$ and $P k D B P \gamma I I$, however, bind only to macaque erythrocytes and not to the Duffy antigen of human erythrocytes [5].

It has been observed that antibodies raised against PkDBPaII could inhibit $P$. knowlesi invasion of human and macque erythrocytes in vitro [6]. Therefore, like PvDBPII for vivax malaria, PkDBPaII may be a candidate vaccine antigen against knowlesi malaria. Any design of vaccine against malaria must take into consideration the nature and genetic polymorphism of the candidate antigen. In a recent study, a high level of genetic diversity was found in the PkDBPoII of 20 P. knowlesi clinical isolates from Peninsular Malaysia [7]. At the amino acid level, 36 haplotypes were identified and these haplotypes could be separated into allele group I and allele group II. In the present study, the PkDBP $\alpha$ II of clinical isolates from the Malaysian states of Sabah and Sarawak in North Borneo was investigated.

\section{Methods}

\section{Blood samples}

The 28 human blood samples used in this study were collected from knowlesi malaria patients at government hospitals in Sabah $(\mathrm{n}=16)$ and Sarawak $(\mathrm{n}=12)$ (Table 1). Confirmation of $P$. knowlesi infection in all the samples was carried out by microscopic examination of Giemsa-stained blood smears and nested polymerase chain reaction (PCR). Ethical approval for the use of human and monkey blood samples in this study were granted by the University of Malaya Medical Centre Ethic Committee (MEC No. 817.18) and the Medical Research Ethic Committee (MREC), Ministry of Health,
Table 1 PkDBPall haplotypes detected in the patient blood samples from Sabah (SBH) and Sarawak (SWK), North Borneo

\begin{tabular}{|c|c|c|}
\hline Blood sample & Origin of sample (district) & Haplotype \\
\hline $\mathrm{SBH} 1$ & Kudat & $\mathrm{H} 37, \mathrm{H} 38, \mathrm{H} 39, \mathrm{H} 40$ \\
\hline $\mathrm{SBH} 2$ & Ranau & $\mathrm{H} 41, \mathrm{H} 42, \mathrm{H} 43$ \\
\hline $\mathrm{SBH} 3$ & Ranau & $\mathrm{H} 44$ \\
\hline $\mathrm{SBH} 4$ & Kota Kinabalu & $\mathrm{H} 45, \mathrm{H} 46$ \\
\hline $\mathrm{SBH} 5$ & Kudat & $\mathrm{H} 47, \mathrm{H} 48, \mathrm{H} 49, \mathrm{H} 50$ \\
\hline $\mathrm{SBH} 6$ & Kota Kinabalu & $\mathrm{H} 47, \mathrm{H} 51, \mathrm{H} 52$ \\
\hline $\mathrm{SBH} 07$ & Kota Kinabalu & $\mathrm{H} 53$ \\
\hline $\mathrm{SBH} 08$ & Kota Kinabalu & $\mathrm{H} 54$ \\
\hline $\mathrm{SBH} 21$ & Kudat & H55 \\
\hline $\mathrm{SBH} 31$ & Ranau & $\mathrm{H} 47$ \\
\hline $\mathrm{SBH} 37$ & Ranau & $\mathrm{H} 47, \mathrm{H} 56, \mathrm{H} 57$ \\
\hline $\mathrm{SBH} 47$ & Kudat & $\mathrm{H} 58$ \\
\hline SBH51 & Kudat & $\mathrm{H} 47, \mathrm{H} 59$ \\
\hline $\mathrm{SBH} 62$ & Ranau & $\mathrm{H} 47, \mathrm{H} 60, \mathrm{H} 61$ \\
\hline SBH68 & Ranau & $\mathrm{H} 47$ \\
\hline SBH71 & Ranau & $\mathrm{H} 62$ \\
\hline SWK01 & Kuching & $\mathrm{H} 63, \mathrm{H} 64$ \\
\hline SWK07 & Kuching & $\mathrm{H} 42$ \\
\hline SWK21 & Kapit & $\mathrm{H} 47$ \\
\hline SWK24 & Kapit & $\mathrm{H} 65, \mathrm{H} 66$ \\
\hline SWK46 & Sri Aman & H67 \\
\hline SWK58 & Kapit & H68 \\
\hline SWK59 & Kapit & H69 \\
\hline SWK72 & Kuching & $\mathrm{H} 47$ \\
\hline SWK76 & Kapit & $\mathrm{H} 70, \mathrm{H} 71$ \\
\hline SWK86 & Kapit & $\mathrm{H} 72$ \\
\hline SWK93 & Kapit & $\mathrm{H} 47, \mathrm{H} 73$ \\
\hline SWK94 & Kapit & $\mathrm{H} 74$ \\
\hline
\end{tabular}

Malaysia (National Medical Research Register ID No. 13079).

\section{Extraction of DNA}

Total DNA of the P. knowlesi was extracted from each blood sample using the QIAGEN Blood DNA Extraction kit (QIAGEN, Hilden, Germany). In each extraction, $100 \mu$ lof blood was used. The extracted DNA was suspended in water to a final volume of $50 \mu \mathrm{l}$.

\section{PCR, cloning and sequencing of the PkDBPall}

The PkDBPoII was amplified by nested PCR using oligonucleotide primers Pk $\alpha$-DBP-F1: 5' -CGCATTTTGAAGG AATCCAC-3' and Pk $\alpha$-DBP-R1: 5'-TGCTAGACTTACC TTCACCT-3' for nest 1 . The primers for the nest 2 reaction were Pk $\alpha$-DBP-F: 5' -TCCTCAAAAGGCGGTGA 
CCATCC-3' and Pk $\alpha$-DBP-R: 5'-ACTGGCTGCCTTAGA TTCAACACCA- $3^{\prime}$. Cycling conditions for nest 1 were as follows: $95^{\circ} \mathrm{C}$ for $4 \mathrm{~min}, 30$ cycles at $95^{\circ} \mathrm{C}$ for $30 \mathrm{sec}, 48^{\circ} \mathrm{C}$ for $30 \mathrm{sec}$, and $72^{\circ} \mathrm{C}$ for $90 \mathrm{sec}$, followed by a 10 -min extension at $72^{\circ} \mathrm{C}$. The amplification for nest 2 was performed using the following cycling profile: $95^{\circ} \mathrm{C}$ for $4 \mathrm{~min}$, 30 cycles at $95^{\circ} \mathrm{C}$ for $30 \mathrm{sec}, 56^{\circ} \mathrm{C}$ for $30 \mathrm{sec}$, and $72^{\circ} \mathrm{C}$ for $90 \mathrm{sec}$, followed by a 10 -min extension at $72^{\circ} \mathrm{C}$. The PCR product with an expected size of 1,053 bp was analysed on a $1 \%$ agarose gel.

\section{Purification of PCR products and DNA cloning}

PCR products were purified by QIAquick PCR purification Kit (QIAGEN, Hilden, Germany) following the manufacturer's instructions. The purified PCR products were then ligated into cloning vector pGEM-T ${ }^{\oplus}$ (Promega Corp, USA) and transformed into Escherichia coli TOP10F'. Plasmids of recombinant clones harbouring the PkDBP $\alpha I$ fragment were sent to a commercial laboratory for DNA sequencing. To detect possibility of multiple haplotypes infecting a patient, plasmids from four to six recombinant clones from each transformation mixture were sequenced.

\section{Analysis of PkDBPall sequences}

Multiple sequence alignment of PkDBP $\alpha I$ was performed using CLUSTAL-Omega programme which was available on-line [8]. Both nucleotide and the deduced amino acid sequences were aligned and analysed. Phylogenetic tree was constructed using the Neighbour Joining method described in MEGA5 [9]. In constructing the phylogenetic tree, bootstrap replicates of 1,000 were used to test the robustness of the tree.

\section{PkDBPall sequence polymorphism analysis}

DnaSP ver. 5.10.00 [10] was used to perform polymorphism analysis on the PkDBPaII sequences. Information such as the number of segregating sites ( $S$ ), haplotype diversity $(\mathrm{Hd})$, nucleotide diversity $(\pi)$, and average number of pair-wise nucleotide differences within the population (K) was generated. The $\pi$ was also calculated on a sliding window of 100 bases, with a step size of 25 bp to estimate the step-wise diversity across PkDBPaII. The rates of synonymous $(K \mathrm{~s})$ and non-synonymous $(K \mathrm{n})$ mutations were estimated and compared by the Z-test $(\mathrm{P}<0.05)$ in MEGA5 using the Nei and Gojobori's method [11] with Jukes and Cantor correction. In the case of purifying (negative) selection, mutations are usually not advantageous so that $K \mathrm{n}$ will be less than $K \mathrm{~s}(K \mathrm{n} / K \mathrm{~s}<1)$. However, in positive selection, nonsynonymous mutations can be advantageous and $K \mathrm{n}$ will exceed $K \mathrm{~s}(K \mathrm{n} / K \mathrm{~s}>1)$. For testing the neutral theory of evolution, Tajima's D [12] and Fu and Li's D and F [13] tests were carried out using DnaSP 5.10.00. In the Fu and Li's tests, P. vivax PvDBPII (GenBank Accession No.
M90466) was used as outgroup. The Wright's $F_{\text {ST }}$ fixation index [14] in DnaSP 5.10.00 was used to measure genetic differentiation between the PkDBPaII of North Borneo and Peninsular Malaysia.

\section{Results}

The nested PCR amplification on the human blood samples produced DNA fragments of $1,053 \mathrm{bp}$ in size. The sequence of each fragment was trimmed to $921 \mathrm{bp}$, as according to the PkDBPaII region described by Singh et al. [15]. The trimmed sequence encoded an amino acid sequence of 307 in length. A final total of 49 sequences (GenBank Accession No. KM926563 - KM926611) were obtained.

DNA sequence analyses were conducted to determine nucleotide diversity and genetic differentiation. The average number of pair-wise nucleotide differences (K) for the PkDBP $\alpha I$ was 11.261. The overall haplotype diversity $(\mathrm{Hd})$ and nucleotide diversity $(\pi)$ for the 49 PkDBPoII sequences were $0.999 \pm 0.004$ and $0.012 \pm$ 0.002 , respectively. Detailed analysis of $\pi$, with a sliding window plot (window length $100 \mathrm{bp}$, step size $25 \mathrm{bp}$ ), revealed diversity ranged from 0.003 to 0.022 . The highest peak of nucleotide diversity was within nucleotide positions 125-250, whereas the most conserved region was within nucleotide positions 625-700 (Figure 1).

Analysis and comparison at the nucleotide level against $P$. knowlesi strain $\mathrm{H}$ as reference sequence (GenBank Accession No. M90466) showed mutations at 160 positions among the North Borneo isolates. Fifty-eight of these mutations were synonymous and 102 were nonsynonymous. To determine whether natural selection contributed to the diversity in the PkDBP $\alpha \mathrm{II}$, the rate of non-synonymous $(K \mathrm{n})$ to synonymous mutations (Ks) was estimated. Kn (0.00900) was found lower than $K \mathrm{~s}$ $(0.02723)$ and the $K \mathrm{n} / K \mathrm{~s}$ ratio was 0.331 , suggesting that purifying (negative) selection may be occurring in the PkDBPaII of the North Borneo isolates. Similarly, the Z test $(K \mathrm{~s}>K \mathrm{n} ; \mathrm{P}<0.05)$ also indicated purifying selection on PkDBPaII. In the tests of departure of neutrality of selection, the Tajima's $\mathrm{D}$ was $-2.459(\mathrm{P}<0.01)$, indicating expansion in population size and/or purifying selection. This is further supported by the Fu and Li's D and F tests statistics $(-3.713$ and -3.917 , respectively; $\mathrm{P}<0.02)$.

Comparison at the amino acid level against the reference $P$. knowlesi strain $\mathrm{H}$ revealed high polymorphism across the PkDBPaII of the North Borneo isolates (Figure 2, bottom half panel). Among the 102 polymorphic sites, 91 were monomorphic change (changed into one amino acid type) and 11 were dimorphic (changed into two amino acid types: positions $4(\mathrm{~N} \rightarrow \mathrm{Q}, \mathrm{T}), 31(\mathrm{~A} \rightarrow \mathrm{S}, \mathrm{T})$, $47(\mathrm{~K} \rightarrow \mathrm{M}, \mathrm{Q}), 65(\mathrm{~T} \rightarrow \mathrm{G}, \mathrm{I}), 95(\mathrm{~N} \rightarrow \mathrm{D}, \mathrm{S}), 121(\mathrm{I} \rightarrow \mathrm{T}, \mathrm{V})$, $122(\mathrm{G} \rightarrow \mathrm{R}, \mathrm{V}), 126(\mathrm{~V} \rightarrow \mathrm{I}, \mathrm{M}), \quad 144 \quad(\mathrm{D} \rightarrow \mathrm{G}, \mathrm{V}), 261$ $(\mathrm{K} \rightarrow \mathrm{E}, \mathrm{R}), 302(\mathrm{H} \rightarrow \mathrm{N}, \mathrm{Y}))$. The PkDBPaII amino acid sequences could be categorized into 38 different 


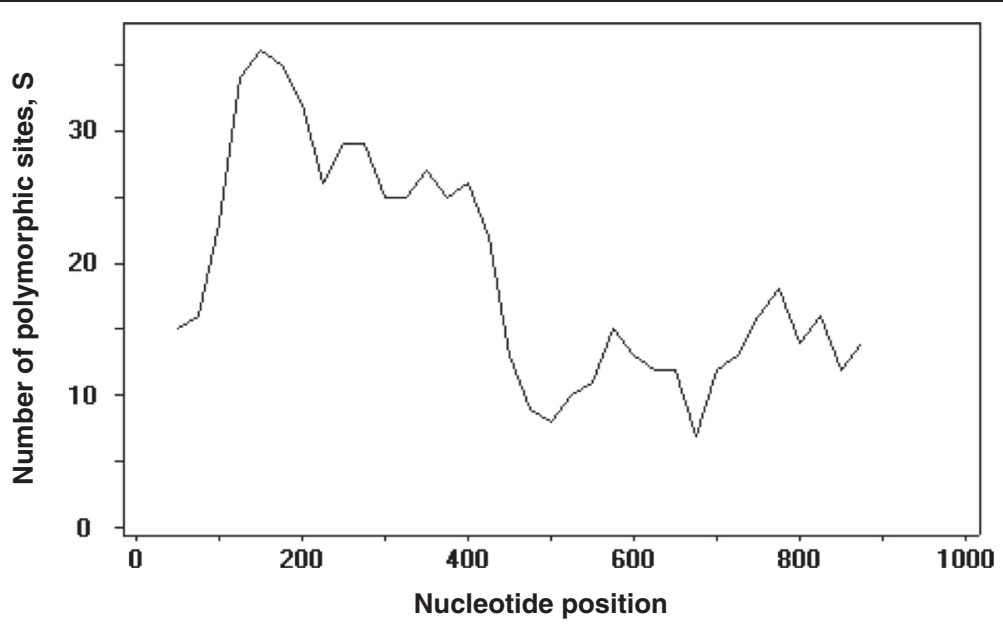

Figure 1 Nucleotide polymorphism in the PkDBPall of Plasmodium knowlesi from North Borneo. Sliding window plot of number of polymorphic sites (S) along the PkDBPall, generated by using DnaSP ver. 5.10 .00 with a window length of $100 \mathrm{bp}$ and step size of $25 \mathrm{bp}$.

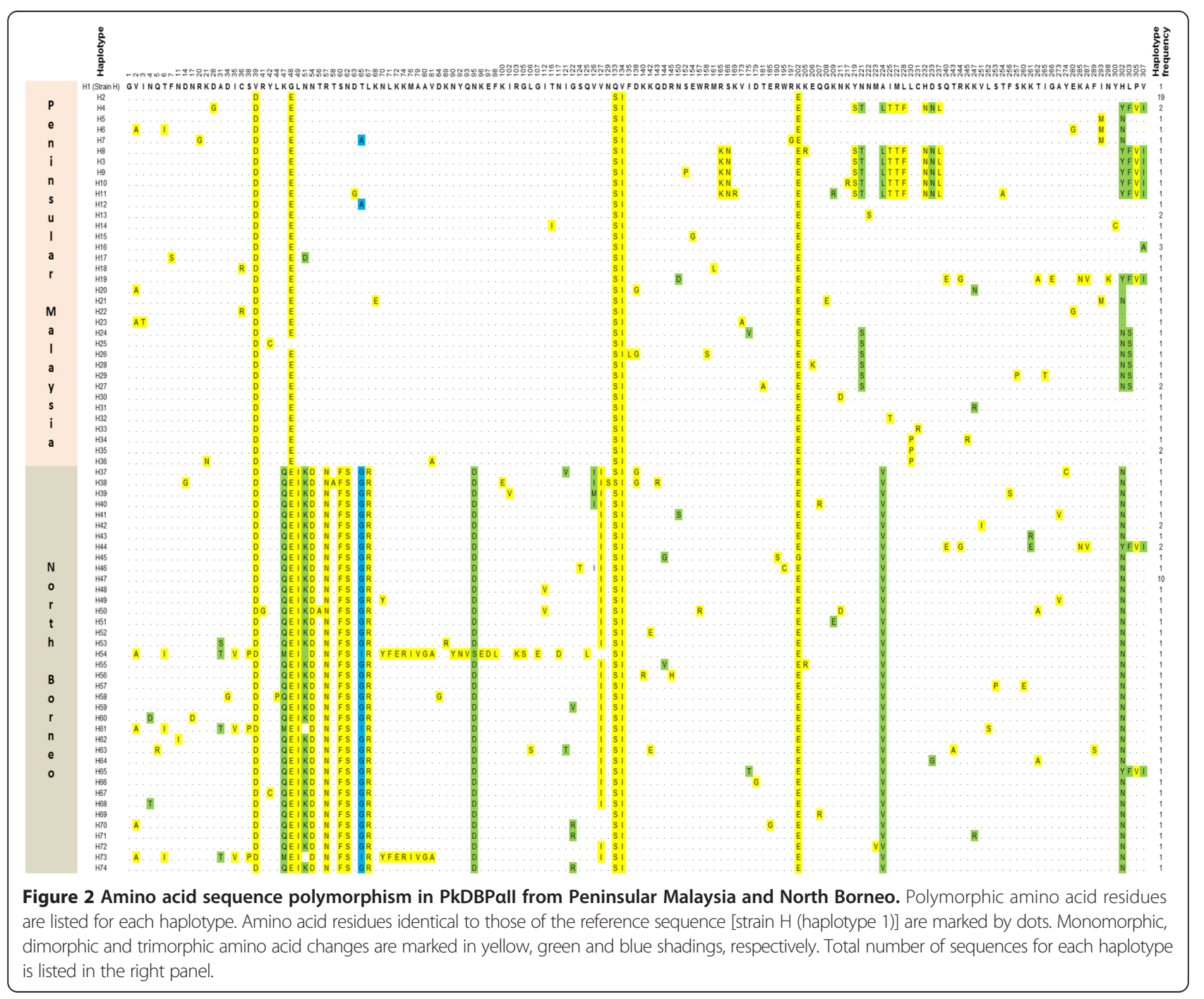


haplotypes (H37-H74) with haplotype 47 having the highest frequency (10/49). Twelve of the 28 blood samples had mixed haplotype infections (Table 1).

A phylogenetic tree comprising these 38 North Borneo and the 36 Peninsular Malaysia haplotypes reported previously [7], showed interesting features (Figure 3). Overall, the haplotypes are still separated into allele group I and allele group II. All the North Borneo haplotypes are in allele group I. However, they form a sub-group which is distinct from allele group I members from Peninsular Malaysia. The Wright's $\mathrm{F}_{\mathrm{ST}}$ value between the PkDBPoII of North Borneo and Peninsular Malaysia was 0.621, indicating high genetic differentiation between these two groups.

\section{Discussion}

The P. knowlesi PkDBPaII plays an essential role in the invasion of the parasite by mediating binding with its corresponding receptor, the Duffy protein receptor for chemokines (DARC) on the surface of erythrocytes [16].
The PkDBPaII elicits immune response in humans and therefore has been suggested to be a vaccine candidate antigen [6]. The genetic diversity and haplotype groups of PkDBP $\alpha I$ among Peninsular Malaysia P. knowlesi clinical isolates were recently reported [7]. The present study found distinct differences in the PkDBP $\alpha \mathrm{II}$ of North Borneo upon comparison with those from Peninsular Malaysia.

Previous studies on $P$. vivax isolates from different geographical regions such as Colombia, South Korea, Papua New Guinea, Thailand, Iran, and Myanmar reported numerous haplotypes and allele groups of PvDBPII [17-22]. Interestingly, some of these PvDBPII haplotypes were grouped with those from outside their geographic origins. For example, haplotypes from Iran were grouped with those from Brazil, Papua New Guines (PNG) and Thailand [21], haplotypes from Myanmar grouped with haplotypes from South Korea [22], and haplotypes from PNG grouped with those from South Korea and Thailand $[18,20]$. This, however, is not observed in the PkDBP $\alpha I$ in

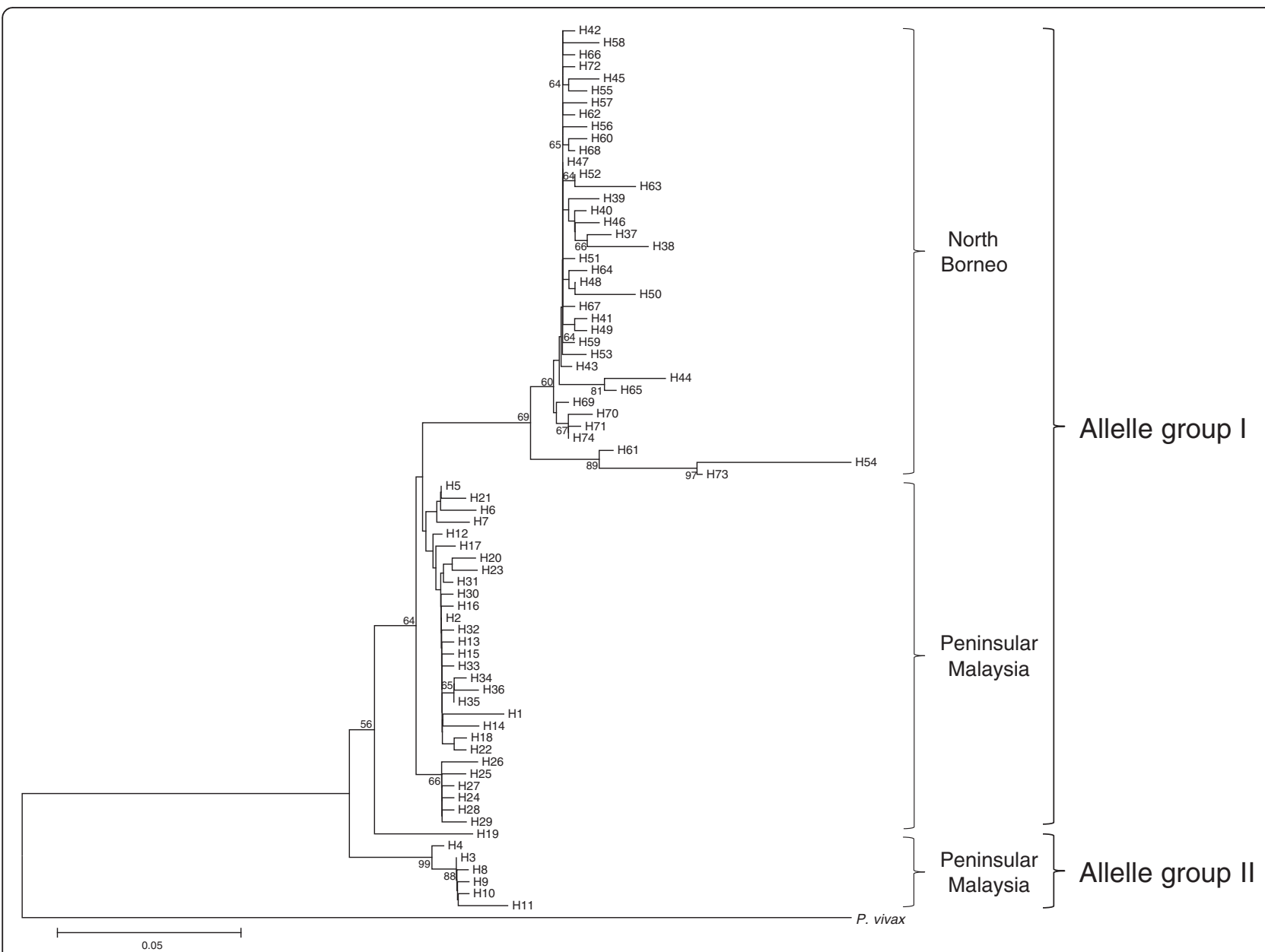

Figure 3 Phylogenetic tree of PkDBPall haplotypes. The Neighbour-Joining method was used to construct the tree, which contains 36 and 38 haplotypes from Peninsular Malaysia (H1-H36) and North Borneo (H37-H74), respectively. Plasmodium vivax PvDBPII was used as outgroup. Numbers at nodes indicate percentage support of 1000 bootstrap replicates. 
the present study. The phylogenetic analysis (Figure 3) showed a sub-group consisted solely of haplotypes from North Borneo, although these haplotypes were still categorized under allele group I. Geographical separation of Borneo Island from Peninsular Malaysia and subsequent genetic drift of the $P$. knowlesi populations may not be the reason for this unique PkDBP $\alpha I I$ separation. This is because other genetic markers studied thus far such as the $P$. knowlesi circumsporozoite protein (csp) gene and the small sub-unit ribosomal rRNA (ssu rRNA) do not display such such geographical-based separation $[1,23,24]$.

The PkDBPoII analysed in this study is based on the region defined by Singh et al. [15]. In their analysis, 12 $\mathrm{C}$ residues (positions 16, 29, 36, 45, 99, 176, 214, 226, $231,235,304,306$ ), which form six disulphide bridges, have been shown to be involved in the folding of PkDBP $\alpha I$ for interaction with DARC. Multiple alignment of the PkDBP $\alpha I I$ amino acid sequences (Additional file 1 ) in this study revealed that these 12 residues were conserved in the PkDBP $\alpha I$ of North Borneo. Apart from these conserved $\mathrm{C}$ residues, the Y94, N95, K96, R103, L168, and I175 residues are required for recognition of DARC on human erythrocytes [15]. The multiple sequence alignment showed high conservation of these residues except at position 95. The $\mathrm{N}$ (asparagine) residue at this position was substituted with the D (aspartic acid) in the PkDBPaII of North Borneo. However, this $\mathrm{N} \rightarrow \mathrm{D}$ substitution may not affect the overall structure and biological function of PkDBPoII, as $\mathrm{N}$ is the amide derivative of $\mathrm{D}$.

The PkDBPaII of North Borneo $(\mathrm{K}=11.261 ; \mathrm{Hd}=$ 0.999; $\pi=0.012)$ was as diverse as that of Peninsular Malaysia $(K=11.736 ; \mathrm{Hd}=0.986 ; \pi=0.013)$. Like the PkDBP $\alpha$ II of Peninsular Malaysia [7], the PkDBP $\alpha I I$ of North Borneo was found to be under purifying (negative) selection. A possible reason for this purifying selection is population expansion of P. knowlesi in Borneo Island, as evident by the Tajima's $\mathrm{D}$, as well as the $\mathrm{Fu}$ and Li's D and F tests statistics. Mitochondrial DNA analysis also suggests recent population expansion of $P$. knowlesi in Southeast Asia [25].

Further evidence of difference between the PkDBPaII of North Borneo and Peninsular Malaysia was shown by the Wright's $\mathrm{F}_{\mathrm{ST}}$ fixation index, which measures population differentiation due to genetic structure [14]. As a rule of thumb, populations with $\mathrm{F}_{\mathrm{ST}}$ values of $>0.25$ are considered highly differentiated. The $\mathrm{F}_{\mathrm{ST}}$ obtained in this study was 0.61 , indicating extremely high genetic difference between the PkDBP $\alpha \mathrm{II}$ of North Borneo and Peninsular Malaysia. The amino acid substitutions in the PkDBPoII, which most likely contribute to this genetic difference, were at positions at positions 47-57, 95 and 224 (Figure 2).

PkDBP $\alpha$ II plays a critical role in the invasion of $P$. knowlesi merozoite into human and monkey erythrocytes.
It is crucial for PkDBP $\alpha I$ to conserve its structure for precise interaction with DARC in the invasion process. The discovery in this study of highly differentiated PkDBP $\alpha I I$ in North Borneo and Peninsular Malaysia may seem puzzling. However, it has been observed that the $P$. vivax PVDBPII is highly diverse, and in some instances within a population of a particular region [26]. DBPII amino acid residues can be variable and these polymorphisms usually map to non-functional regions of the protein, therefore may serve as a mechanism of immune evasion for the parasite. In such a mechanism, polymorphic residues near the binding site escape binding of host inhibitory antibodies. This protects the crucial functional site on the interacting DBPII domain.

A recent phylogenetic study on the relationships of Macaca fascicularis, the natural monkey host of $P$. knowlesi, showed a clear separation between Borneo's and Peninsular Malaysia's populations [27]. This phylogeny was based on the cytochrome $b$ gene sequences of the monkeys. It is, therefore, worthwhile in future studies to determine whether a similar genetic separation occurs in the DARC of the monkey populations, and to associate it with the PkDBPaII haplotype groups observed in this study.

\section{Conclusions}

This study is the first to report the genetic diversity and natural selection of PkDBPaII of P. knowlesi from Borneo Island. The PkDBPoII haplotypes found in this study were distinct from those from Peninsular Malaysia. This difference may not be attributed to geographical separation because other genetic markers studied thus far such as the $P$. knowlesi circumsporozoite protein gene and small subunit ribosomal RNA do not display such differentiation. Immune evasion may possibly be the reason for the differentiation.

\section{Additional file}

Additional file 1: Full amino acid sequence alignment of PkDBPall from Peninsular Malaysia and North Borneo. Amino acid residues identical to those of the reference sequence (strain $\mathrm{H}$ ) are indicated by dots. The twelve conserved cysteine $(C)$ residues are marked in yellow. The conserved Y94, N95, K96, R103, L168 and 1175 residues required for recognition of DARC on human erythrocytes are highlighted in green. Note that the $\mathrm{N} 9$ residue was substituted by D95 in the North Borneo sequences.

\section{Competing interests}

The authors declare that they have no competing interests.

Authors' contributions

MYF and YLL designed the study and supervised the study process. SAAR and RY performed all the experiments and analysed the sequence data. MYF performed sequence and phylogenetic analyses. MYF and YLL wrote the manuscript. All authors read and approved the final manuscript. 


\section{Acknowledgements}

This study was supported by the UM High Impact Research Grant UM-MOHE UM.C/625/1/HIR/MOHE/MED/09 from the Ministry of Education, Malaysia. We thank the participating hospitals in Sabah and Sarawak for providing patient blood samples.

Received: 3 December 2014 Accepted: 10 February 2015

Published online: 21 February 2015

\section{References}

1. Singh B, Kim Sung L, Matusop A, Radhakrishnan A, Shamsul SS, Cox-Singh J, et al. A large focus of naturally acquired Plasmodium knowlesi infections in human beings. Lancet. 2004;363:1017-24.

2. Galinski MR, Barnwell JW. Monkey malaria kills four humans. Trends Parasitol. 2009;25:200-4

3. Yusof R, Lau YL, Mahmud R, Fong MY, Jelip J, Ngian HU, et al. High proportion of knowlesi malaria in recent malaria cases in Malaysia. Malar J. 2014;13:168.

4. Gaur D, Mayer DC, Miller LH. Parasite ligand-host receptor interactions during invasion of erythrocytes by Plasmodium merozoites. Int J Parasitol. 2004;34:1413-29.

5. Adams JH, Sim BKL, Dolan SA, Fang X, Kaslow DC, Miller LH. A family of erythrocyte binding proteins of malaria parasites. Proc Natl Acad Sci U S A. 1992;89:7085-9.

6. Singh AP, Puri SK, Chitnis CE. Antibodies raised against receptor-binding domain of Plasmodium knowlesi Duffy binding protein inhibit erythrocyte invasion. Mol Biochem Parasitol. 2002;121:21-31.

7. Fong MY, Lau YL, Chang PY, Anthony CN. Genetic diversity, haplotypes and allele groups of Duffy binding protein (PkDBPall) of Plasmodium knowlesi clinical isolates from Peninsular Malaysia. Parasit Vectors. 2014;7:161.

8. Clustal Omega, a multiple sequence alignment program available online at http://www.ebi.ac.uk/Tools/msa/clustalo.

9. Tamura K, Peterson D, Peterson N, Stecher G, Nei M, Kumar S. MEGA5: Molecular evolutionary genetics analysis using maximum likelihood, evolutionary distance, and maximum parsimony methods. Mol Biol Evol. 2011;28:2731-9.

10. Librado P, Rozas J. DnaSP v5: a software for comprehensive analysis of DNA polymorphism data. Bioinformatics. 2009;25:1451-2.

11. Nei M, Gojobori T. Simple methods for estimating the numbers of synonymous and nonsynonymous nucleotide substitutions. Mol Biol Evol. 1986;3:418-26.

12. Tajima F. Statistical method for testing the neutral mutation hypothesis by DNA polymorphism. Genetics. 1989;123:585-95.

13. Fu YX, Li WH. Statistical tests of neutrality of mutations. Genetics. 1993;133:693-709.

14. Wright S. The genetical structure of populations. Ann Eugen. 1951:15:323-54

15. Singh SK, Hora R, Belrhali H, Chitnis CE, Sharma A. Structural basis for Duffy recognition by the malaria parasite Duffy-binding-like domain. Nature. 2006:439:741-4

16. Demogines A, Truong KA, Sawyer SL. Species-specific features of DARC, the primate receptor for Plasmodium vivax and Plasmodium knowlesi. Mol Biol Evol. 2012;29:445-9.

17. Ampudia E, Patarroyo MA, Patarroyo ME, Murillo LA. Genetic polymorphism of the Duffy receptor binding domain of Plasmodium vivax in Colombian wild isolates. Mol Biochem Parasitol. 1996;78:269-72.

18. Ju HL, Kang JM, Moon SU, Bahk YY, Cho PY, Sohn WM, et al. Genetic diversity and natural selection of Duffy binding protein of Plasmodium vivax Korean isolates. Acta Trop. 2013;125:67-74.

19. Xainli J, Adams JH, King CL. The erythrocyte binding motif of Plasmodium vivax Duffy binding protein is highly polymorphic and functionally conserved in isolates from Papua New Guinea. Mol Biochem Parasitol. 2000;111:253-60

20. Gosi P, Khusmith S, Khalambaheti T, Lanar DE, Schaecher KE, Fukuda MM, et al. Polymorphism patterns in Duffy-binding protein among Thai Plasmodium vivax isolates. Malar J. 2008;7:112.

21. Babaeekho L, Zakeri S, Djadid ND. Genetic mapping of the Duffy binding protein (DBP) ligand domain of Plasmodium vivax from unstable malaria region in the Middle East. Am J Trop Med Hyg. 2009:80:112-8.

22. Ju HL, Kang JM, Moon SU, Kim JY, Lee HW, Lin K, et al. Genetic polymorphism and natural selection of Duffy binding protein of Plasmodium vivax Myanmar isolates. Malar J. 2012;11:60.
23. Vythilingam I, Noorazian YM, Huat TC, Jiram Al, Yusri YM, Azahari AH, et al Plasmodium knowlesi in humans, macaques and mosquitoes in peninsular Malaysia. Parasit Vectors. 2008;1:26.

24. Jiram Al, Vythilingam I, NoorAzian YM, Yusof YM, Azahari AH, Fong MY. Entomologic investigation of Plasmodium knowlesi vectors in Kuala Lipis, Pahang. Malaysia Malar J. 2012;11:213.

25. Lee KS, Divis PC, Zakaria SK, Matusop A, Julin RA, Conway DJ, et al. Plasmodium knowlesi: reservoir hosts and tracking the emergence in humans and macaques. PLoS Pathog. 2011;7:e1002015.

26. de Sousa TN, Kano FS, de Brito CF, Carvalho LH. The Duffy binding protein as a key target for a Plasmodium vivax vaccine: lessons from the Brazilian Amazon. Mem Inst Oswaldo Cruz. 2014;109:608-17.

27. Abdul-Latiff MAB, Ruslin F, Vun VF, Mohd-Hashim A, Rovie-Ryan tر Abdul-Patah P, et al. Phylogenetic relationships of Malaysia's long-tailed macaques, Macaca fascicularis, based on cytochrome $b$ sequences. ZooKeys. 2014;407:121-40

\section{Submit your next manuscript to BioMed Central and take full advantage of:}

- Convenient online submission

- Thorough peer review

- No space constraints or color figure charges

- Immediate publication on acceptance

- Inclusion in PubMed, CAS, Scopus and Google Scholar

- Research which is freely available for redistribution 